\title{
A method for in vivo detection of abnormal subepidermal tissues based on dielectric properties
}

\author{
Liang Zhang ${ }^{\mathrm{a}}$, Peiguo Liu ${ }^{\mathrm{b}}$, Xiuzhen Dong ${ }^{\mathrm{c}}$, Dongming Zhou ${ }^{\mathrm{b}, *}$ and Xuetao Shi ${ }^{\mathrm{c}, *}$ \\ ${ }^{a}$ College of Science, National University of Defense Technology, Changsha, Hunan 410073, P.R. \\ China \\ ${ }^{b}$ State Key Laboratory of Complex Electromagnetic Environmental Effects on Electronics \& \\ Information System, National University of Defense Technology, Changsha, Hunan 410073, P.R. \\ China \\ ${ }^{c}$ Faculty of Biomedical Engineering, Fourth Military Medical University, Xi'an, Shaanxi 710032, P.R. \\ China
}

\begin{abstract}
In this paper, a convenient and noninvasive scanning imaging method using microwave frequency band for abnormal subepidermal tissues detection is proposed in the aim to improve diagnosis and prognosis in clinical environments. This method is based on the reflective detection technology with coaxial probe that is used to measure the dielectric properties of tissues. An improved equivalent circuit and simulated annealing algorithm (SA) were used in this work to analyze the dielectric properties of tissues. Computational simulations incorporating a simplified model of subcutaneous hemorrhage described in this work were used to evaluate this method. The dielectric properties data of tissues in the model of simulation is derived from the literature. The simulation results demonstrated the potential of this method to detect abnormal subepidermal tissues conveniently and expose them in the image accurately.
\end{abstract}

Keywords: Coaxial probe, dielectric properties, scanning imaging, simulated annealing, subepidermal tissues

\section{Introduction}

The improvement of medical detection technology has always been the pursuit of scientist for enhanced diagnosis and prognosis in clinical environments. Currently detection technologies based on physical properties, such as the computed tomography (CT) and magnetic resonance imaging (MRI) are predominant in the field of medical detection. Particularly, electrical and electromagnetic detection methods with noninvasive ability but high penetrability is becoming more popular and attracted more

\footnotetext{
${ }^{*}$ Corresponding authors: Dongming Zhou, State Key Laboratory of Complex Electromagnetic Environmental Effects on Electronics \& Information System, National University of Defense Technology, Changsha, Hunan 410073, P.R. China. Tel.: +860731 84576219; Fax: +86073184576219; E-mail: dmzhou@nudt.edu.cn.

Xuetao Shi, Faculty of Biomedical Engineering, Fourth Military Medical University, Xi'an, Shaanxi 710032, P.R. China. Tel.: +86 029 84776397; Fax: +86029 84776397; E-mail: shixuetao@fmmu.edu.cn.
}

0959-2989/14/\$27.50 @ 2014 - IOS Press and the authors. 
attentions from researchers. Take the electrical measurement as an example: Halter investigated the possibility of differentiating normal and malignant prostate tissue using impedance spectra [1]; S. Abdalla investigated the low-frequency dielectric properties of blood and hence suggested new diagnostic and therapeutic methods for blood disorders [2]. Besides, imaging technology based on electromagnetism is also under rapid development. Microwave imaging technology was studied for the detection of breast cancer [3]. Semenov also developed reconstructed approaches for the quality of microwave images [4].

However, existing means in medical detection are still incompetent to many diseases including diseases under the skin. Subcutaneous hemorrhage is one of the symptoms in the subepidermal tissue which usually indicates the injury of blood vessel. Skin cancer is one of the most common cancers in the world, and melanoma has the highest mortality rate of all the skin cancer subtypes [5]. The conventional diagnosis of these diseases mainly depends on the histopathological examination. However, histopathological examination is far from ideal due to its inconvenience. On the other hand, new methods such as Magnetic Resonance Imaging (MRI) still have disadvantages on high cost in practice. Therefore, there is a continuing need for developing and improving new detection technology for clinical diagnosis.

This study presents a surface scanning imaging method for the detection of abnormal tissues under the skin. This method is based on the reflective detection technology using coaxial probe in microwave frequency band. An improved equivalent circuit and Simulated Annealing (SA) algorithm were used in this work to calculate the dielectric properties of tissues. The simplified model of subcutaneous hemorrhage, built according to the results from Gabriel et al. [6], was used in the simulation. The simulation results demonstrated the potential of this method to detect the abnormal subepidermal tissues and expose them in the image.

\section{Methods}

\subsection{Improved equivalent circuit}

The coaxial probe is commonly utilized in measuring the dielectric properties of biological tissues $[7,8]$. In this work, the sensor of detection was modified accordingly from the open-ended coaxial probe whose inner conductor is slightly longer than the outer conductor, as shown in Figure 1(a). This modified setup can help increase the depth of detection.

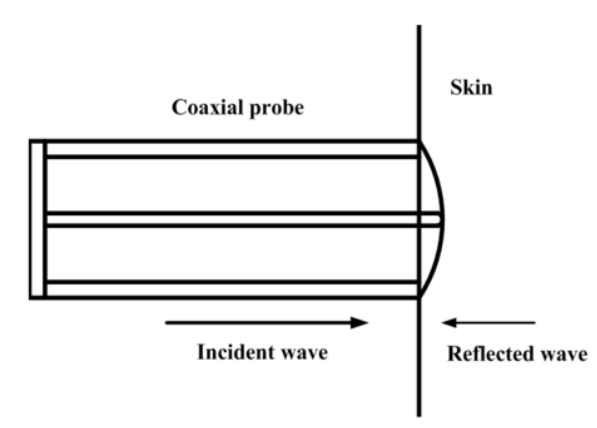

(a)

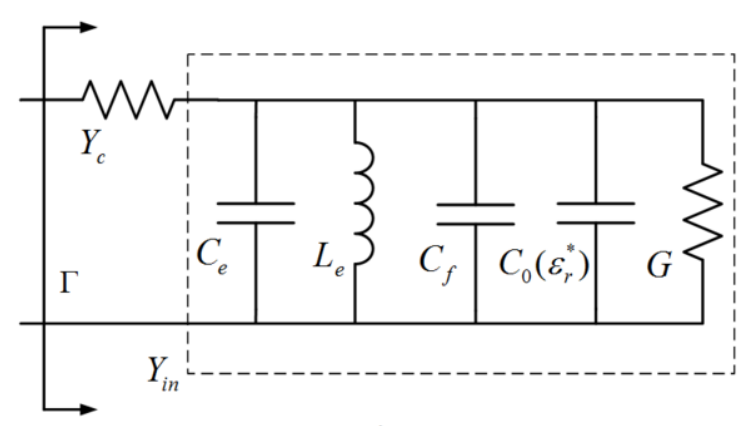

(b)

Fig. 1. The fixture and equivalent circuit of sensor: (a) The fixture of sensor; (b) The equivalent circuit. 
When the sensor is terminated by the skin, the reflection coefficient $\Gamma$ of detection fixture is measured at the desired frequency. This fixture could be taken as the transmission line. The improved equivalent circuit of this transmission line as shown in Figure 1(b) was used to calculate the dielectric properties of biological tissue [9].

The reflection coefficient $\Gamma$ is related to the characteristic admittance of sensor $Y_{c}$ and the terminal input admittance of sensor $Y_{\text {in }}$ which contains the dielectric information of the skin. According to the literature [10], the terminal input admittance of sensor $Y_{\text {in }}$ can be calculated as:

$$
Y_{\text {in }}=Y_{c} \frac{1-\Gamma}{1+\Gamma}
$$

According to Figure 1(b), the terminal input admittance of sensor $Y_{\text {in }}$ can also be expressed as:

$$
Y_{i n}=j \omega C_{f}\left(\varepsilon_{r}^{\prime}\right)+j \omega C_{0} \varepsilon_{r}+G\left(\varepsilon_{r}^{\prime}, \sigma, \omega\right)+j b_{0}
$$

$\mathrm{C}_{\mathrm{f}}$ is taken as the dispersive capacitance of the probe is relative to $\varepsilon_{\mathrm{r}}^{\prime} . \mathrm{C}_{0}$ is the terminal capacitance of detection fixture when the coaxial probe is terminated by air. $\mathrm{G}\left(\varepsilon_{\mathrm{r}}^{\prime}, \sigma, \omega\right)$ represents the radiation ability of the coaxial opening. $b_{0}$ is the error term. All parameters were described in detail in the previous study [9].

\subsection{The simulated annealing (SA) algorithm}

According to Eq. (2), the dielectric properties of biological tissues $\varepsilon_{\mathrm{r}}^{*}\left(\varepsilon_{\mathrm{r}}, \sigma\right)$ could not be calculated directly [9]. Therefore, simulated annealing (SA) algorithm was adopted to calculate $\varepsilon_{\mathrm{r}}^{*}\left(\varepsilon_{\mathrm{r}}, \sigma\right)$ from Eq. (2). First, the random initialized solutions $\left(\varepsilon_{\mathrm{r} 0}, \sigma_{0}\right)$ was given. Then new solutions $\left(\varepsilon_{\mathrm{r} j}, \sigma_{\mathrm{j}}\right)$ were searched randomly and two groups of solutions were compared by the cost function $f\left(\varepsilon_{\mathrm{r}}^{\prime}, \sigma\right)$. The good solutions were kept and then the procedure continued until the conditions were met. The detailed description of this algorithm was described in [9].

\subsection{The factors of imaging}

When the sensor took regular movement on the surface of skin, the dielectric properties of biological tissues of each sample position $\varepsilon^{*}{ }_{r}(i, j)$ were calculated according to Eqs. (1) and (2). As a result, the dielectric properties of biological tissues matrix $\varepsilon_{\mathrm{r}}^{*}$ could be derived from reflection coefficient matrix $\Gamma$ :

$$
\boldsymbol{\Gamma}=\left[\begin{array}{ccc}
\Gamma(1,1) & \ldots & \Gamma(1, n) \\
\ldots & \ldots & \ldots \\
\Gamma(n, 1) & \ldots & \Gamma(n, n)
\end{array}\right] \rightarrow \boldsymbol{\varepsilon}_{\mathbf{r}}^{*}=\left[\begin{array}{ccc}
\varepsilon_{r}^{*}(1,1) & \ldots & \varepsilon_{r}^{*}(1, n) \\
\ldots & \ldots & \ldots \\
\varepsilon_{r}^{*}(n, 1) & \ldots & \varepsilon_{r}^{*}(n, n)
\end{array}\right]
$$




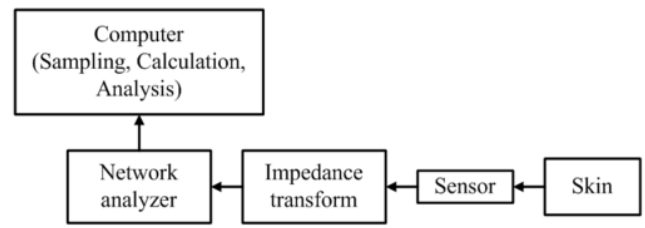

Fig. 2. The system structure diagram of the scanning imaging.

$\mathrm{n}$ is the number of the samples based on position. The dielectric properties matrix $\boldsymbol{\varepsilon}_{\mathrm{r}}^{*}$ could be used for the impedance imaging. If there is any abnormal subepidermal tissue, $\varepsilon_{\mathrm{r}}^{*}(\mathrm{i}, \mathrm{j})$ would be changed and appear in the image.

Figure 2 gives the system structure diagram of the scanning imaging. The sensor was connected to the network analyzer by impedance transform module that is used to achieve the impedance match between sensor and network analyzer. When the detection began, the network analyzer was mainly used to measure the reflection coefficient $\Gamma$ from the sensor in real time. The computer was used to take samples, transform $\Gamma(\mathrm{i}, \mathrm{j})$ into $\varepsilon_{\mathrm{r}}^{*}(\mathrm{i}, \mathrm{j})$ and build images of subepidermal tissues.

\section{Data generation and simulation settings}

\subsection{Simplified model of subcutaneous hemorrhage}

The verification of this detection method was simulated using the simplified model of subcutaneous hemorrhage. Blood was treated as the single abnormal tissue under the skin and used to simulate the symptom of subcutaneous hemorrhage. Critical parameters for the detection including the dielectric properties of skin and blood were derived from Gabriel's four-pole Debye model [6]:

$$
\varepsilon_{r}^{*}=\varepsilon_{\infty}+\sum_{i=1}^{4} \frac{\Delta \varepsilon_{i}}{1+j \omega \tau_{i}}+\frac{\sigma_{s}}{j \omega \varepsilon_{0}}
$$

where $\varepsilon_{\infty}$ is the high frequency permittivity and $\sigma_{\mathrm{s}}$ is the static conductivity. $\tau$ is the relaxation time, and $\alpha$ is the relaxation factor. With the model parameters of skin and blood provided in Gabriel's study [6], the corresponding dielectric properties can be calculated by Eq. (4).

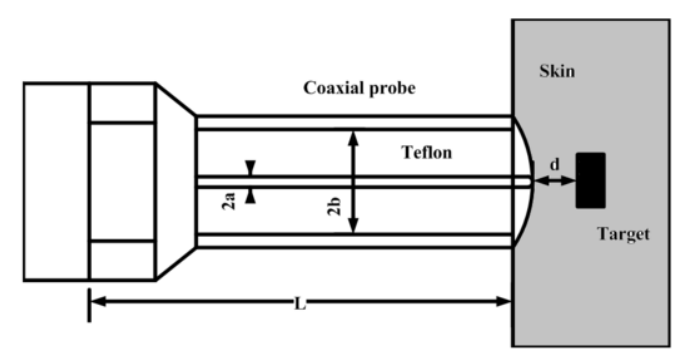

Fig. 3. The diagram of simulation settings. 


\subsection{Simulation settings}

Figure 3 shows the diagram of simulation settings. Skin was taken as the background material which was set up as a single cylinder with a radius of $20 \mathrm{~mm}$ and a thickness of $5 \mathrm{~mm}$. Blood was taken as the abnormal target which was set up as a cube with the size of $4 \mathrm{~mm} \times 4 \mathrm{~mm} \times 1.5 \mathrm{~mm}$. The depth of the target in the skin $\mathrm{d}$ was set to be $1.5 \mathrm{~mm}$. These settings make the model more realistic so that it can keep the target below the epidermis where the blood vessels gather.

The dimensions of the sensor in the simulation were set as follows: the outer conductor was set to be $\mathrm{b}$ in radius. The space between the inner conductor and outer conductor was filled with Teflon. The opening radius of probe was $b=4 \mathrm{~mm}$ and inner radius was $\mathrm{a}=1.2 \mathrm{~mm}$. The terminal of inner conductor was set to be $1 \mathrm{~mm}$ longer than outer conductor, forming a spherical surface to reduce the friction during scanning. The length of the probe $\mathrm{L}$ was set up to be $30 \mathrm{~mm}$. The scanning area was set up as a square with the size of $20 \mathrm{~mm} \times 20 \mathrm{~mm}$. The simulation was achieved in microwave software CTS.

When the sensor touched the skin, the tissue would be depressed due to the longer inner conductor. The longer inner conductor was used to expand the effect area of electromagnetic field and increase the depth of detection. But it could also prevent the outer conductor unable to contact with the skin properly if the inner conductor was set to be too long. In this study, inner conductor was set to be 1 mm longer in order to keep proper contact between the outer conductor and the skin. On the other hand, the gap between the sensor and skin may interfere with probe detection. In our simulation, we estimated and considered the worst scenario that the area of gap was maximal where the outer conductor just had contact with the skin properly, as shown in Figure 3. In fact, this factor is hard to analyze in the actual measurement.

Several other parameters were set according to the requirement of this study, as described below:

(1) Frequency: The working frequency of detection was set to be $1.4 \mathrm{GHz}, 3.6 \mathrm{GHz}$ and $4.8 \mathrm{GHz}$.

(2) Normalized Imaging Factor: The dielectric properties of biological tissues matrix $\boldsymbol{\varepsilon}_{\mathbf{r}}^{*}$ was defined as the imaging factor which was used to build the image of scanning results. It was normalized prior to comparisons across different imaging measurements:

$$
\begin{aligned}
& \boldsymbol{\varepsilon}_{\mathbf{r} n o r}^{\prime}=\left(\frac{\boldsymbol{\varepsilon}_{\mathbf{r}}^{\prime}}{\min \left(\left|\varepsilon_{\mathbf{r}}^{\prime}\right|\right.}\right) \\
& \boldsymbol{\sigma}_{\text {nor }}=\left(\frac{\boldsymbol{\sigma}}{\min (|\boldsymbol{\sigma}|)}\right)
\end{aligned}
$$

\section{Results}

After CST calculated the reflection coefficients of sensor, the dielectric properties of tissues on measurement would be calculated and changed into images. The images at different frequencies of subcutaneous hemorrhage model reconstructed previously were presented in Figure 4. Figures 4(a)-4(c) are images based on the permittivity $\varepsilon_{\mathrm{r}}$ of biological tissues at $1.4 \mathrm{GHz}, 3.6 \mathrm{GHz}$ and $4.8 \mathrm{GHz}$. Figures 4(d)-4(f) are images based on the conductivity $\sigma$ of biological tissues at $1.4 \mathrm{GHz}, 3.6 \mathrm{GHz}$ and 4.8 $\mathrm{GHz}$. 


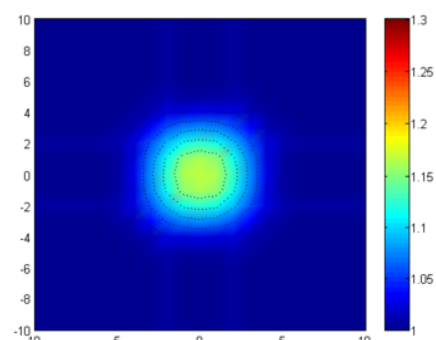

(a)

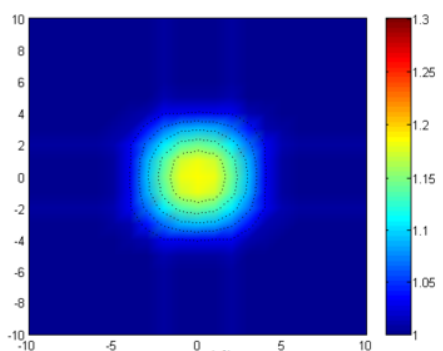

(d)

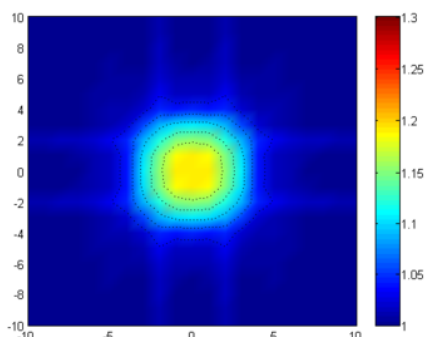

(b)

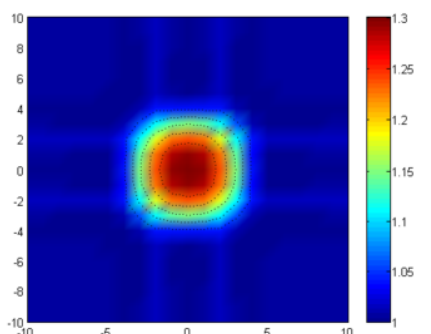

(e)

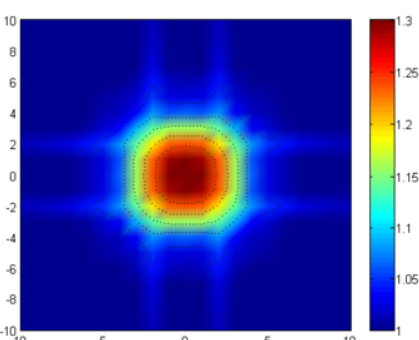

(c)

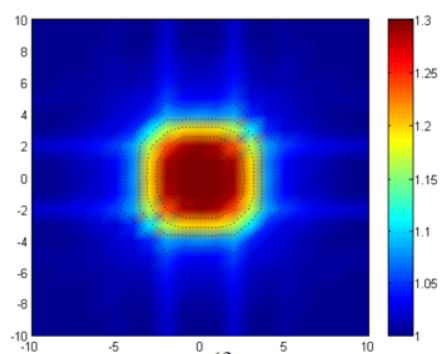

(f)

Fig. 4. The images at different frequencies of subcutaneous hemorrhage model. (a)-(c) images based on the permittivity $\varepsilon$ 'r at $1.4 \mathrm{GHz}, 3.6 \mathrm{GHz}$ and $4.8 \mathrm{GHz}$. (d)-(f) images based on the conductivity $\sigma$ at $1.4 \mathrm{GHz}, 3.6 \mathrm{GHz}$ and $4.8 \mathrm{GHz}$.

As shown in Figure 4, this method presented the subcutaneous hemorrhage simplified model well at three working frequencies. The best result was achieved at $4.8 \mathrm{GHz}$ that the target was reconstructed with the most details, whether based on permittivity $\varepsilon_{\mathrm{r}}^{\prime}$ or conductivity $\sigma$. This is because the resolving power of target detection is higher as the frequency increases. On the other hand, the permittivity of biological tissues in our working frequencies is very large. This could lead to a scenario that the sensitivity of detection to the tissues with different permittivity was not high enough when the depth of detection was small. As a result, the images of detection based on conductivity $\sigma$ in Figures 4(d)-4(f) were better than permittivity $\varepsilon_{\mathrm{r}}$ in Figures $4(\mathrm{a})-4(\mathrm{c})$.

\section{Discussion}

From Figure 4, it is clear that the result of detection at high frequency was better than the result of detection at low working frequency. However, the effect of water contained in biological tissues which was not fully incorporated into the model would be more prominent as the working frequency increases. This would reduce the penetrability of the sensor which determines the depth of detection and sensitivity of detection to the target. As a result, the working frequency should not be too high. In practice, the frequency was usually kept below $3 \mathrm{GHz}$ where the dielectric spectrum of biological tissue was between the $\beta$ and $\gamma$ dispersions during measurement [11].

This scanning imaging method uses the coaxial probe as the detection sensor that could take advantage of a wide frequency band plus smaller design dimensions. Another important advantage is the sensor is terminated with the skin once the detection is initiated. This fixture could decrease the influence of noise in the space, providing a quieter environment compared to other detection methods such as the microwave imaging. 
The sampling rate of the system could also affect to the spatial resolution of the imaging. In this study, the interval of scanning is equal to the sampling rate. On the other hand, the sensor of detection could also be replaced by a detection array that consists of many probes arraying in sequential order. This technology can be applied in areas such as phased array, synthetic aperture as well as for improving the result of detection, while how to improve the resolution of measurement is still a challenge that needs further studies in the future.

\section{Conclusion}

A surface scanning imaging method is presented in this paper for the detection of abnormal tissues under the skin. This method is based on the reflective detection technology using coaxial probe within microwave frequency band. An improved equivalent circuit and simulated annealing (SA) algorithm were used in this work to calculate the dielectric properties of tissues. According to the results of computational simulations incorporating the simplified model of subcutaneous hemorrhage, this method can successfully detect abnormal subepidermal tissues and expose them accurately in the image. It is necessary to set the working frequency not too high in order to reduce the influence of water contained in biological tissues. In future studies, resolution improvement should be prioritized and more emerging technologies such as detection sensor array should be considered.

\section{Acknowledgment}

This study was supported by the Research Fund for the Doctoral Program of Higher Education of China under grant 20114307110022, the National Science and Technology Pillar Program of China under grant 2012BAI19B01 and the National Natural Science Foundation of China under grant 61372029 .

\section{References}

[1] R.J. Halter, A. Hartov, J.A. Heaney et al., Electrical impedance spectroscopy of the human prostate, IEEE Trans. on Biomedical Engineering 54 (2007), 1321-1327.

[2] S. Abdalla, Low frequency dielectric properties of human blood, IEEE Trans. on Nanobioscience 10 (2011), 113-120.

[3] L. Kanelovitch, Y. Itzchak, A. Rundstein et al., Biologically derived companding algorithm for high dynamic range mammography images, IEEE Trans. on Biomedical Engineering 60 (2013), 2253-2261.

[4] S.Y. Semenov, A.E. Bulyshev, A. Abubakar et al., Microwave-tomographic imaging of the high dielectric-contrast objects using different image-reconstruction approaches, IEEE Trans. on Microwave Theory and Techniques 53 (2005), 2284-2294.

[5] D.J. Schwartzentruber, D.H. Lawson, J.M. Richards et al., Gp100 peptide vaccine and interleukin-2 in patients with advanced melanoma, New England Journal of Medicine 364 (2011), 2119-2127.

[6] S. Gabriel, R. Lau and C. Gabriel, The dielectric properties of biological tissues: III. Parametric models for the dielectric spectrum of tissues, Physics in Medicine and Biology 41 (1996), 2271-2293.

[7] C. Gabriel, T.Y.A. Chan and E.H. Grant, Admittance models for open ended coaxial probes and their place in dielectric spectroscopy, Physics in Medicine and Biology 39 (1994), 2183-2200.

[8] D. Popovic, L. McCartney, C. Beasley et al., Precision open-ended coaxial probes for in vivo and ex vivo dielectric spectroscopy of biological tissues at microwave frequencies, IEEE Trans. on Microwave Theory and Techniques 53 (2005), 1713-1722.

[9] Liang Zhang, Xuetao Shi, Fusheng You et al., Improved circuit model of open-ended coaxial probe for measurement of the biological tissue dielectric properties between megahertz and gigahertz, Physiol. Meas. 34 (2013), N83-N96. 
[10] F.M. Ghannouchi and R.G. Bosisio, Measurement of microwave permittivity using a six-port reflectometer with an open-ended coaxial probe, IEEE Trans. on Instrumentation and Measurement 38 (1989), 505-508.

[11] C. Gabriel, S. Gabriel and E. Corthout, The dielectric properties of biological tissues: I. Literature survey, Physics in Medicine and Biology 41 (1996), 2231-2249. 